\title{
Optimisation of the bidding strategy for wind power trading
}

\author{
David I. Hamilton, David McMillan, Victoria M. Catterson \\ Electronic and Electrical Engineering \\ University of Strathclyde \\ Glasgow, United Kingdom \\ david.hamilton@strath.ac.uk
}

\begin{abstract}
The optimal bidding strategy for trading electricity from a wind farm is not always clear. This paper outlines a method for predicting whether the market will be long or short and uses this information to select the best quantile regression for the current market conditions. Results from a simulation with a $2.5 \mathrm{MW}$ turbine produced a savings of over $£ 1000$ over a three month period compared to using only a P50 forecaster.
\end{abstract}

Index Terms-Wind energy, energy markets, quantile regression,

\section{INTRODUCTION}

One problem in the wind power industry is how to deliver an optimal trading strategy for the electricity generated from a wind farm; this problem will only become more pronounced as the penetration of wind energy increases.

The current literature on this matter skews towards risk averse strategies (Galloway, Bell, McDonald, \& Siewierski, 2006) (Matevosyan \& Soder, 2006), indicating that a generator should contract low and accept whatever price is offered for over generation. As more wind farms are connected to the transmission system this strategy becomes less desirable as the risk of curtailment increases, if a generator is over their contracted volume and there is too much power on the system (Gardner \& Papadopoulos, 2012).

If the state of the electricity market could be predicted then a generator would know whether to contract a cautious or optimistic level. (Jonsson, Pinson, Nielson, \& Madsen, 2014) employed a Holt-Winters model to make a day ahead forecast of the state of the Nord Pool Elspot market with good results. This paper follows on from the ideas explored by these authors, applying them to the UK energy market using a different model to predict the state of the market.

The Nord Pool is divided into two markets: the Elspot and the Elbas. The Elspot is a day ahead market; trading for the next day is finalised at noon and the price of electricity for each hour is calculated. The Elbas is an intraday market where trades can be made up to one hour before delivery. The
UK electricity market is combination of these two where the majority of electricity is traded well in advance of the settlement period but trading can occur up to one hour before delivery.

This paper uses data from the UK electricity market to forecast the state of the market and uses this information to select from a range of quantile regressions that best reflect the current optimal trading strategy. This is shown to be financially beneficial over a more cautious strategy.

\section{MARKET OVERVIEW}

Electricity in the UK is traded in thirty minute settlement periods. Generators are required to submit the level of expected generation traded from each of their assets one hour before the start of a settlement period.

Parties that wish to trade electricity in the UK are required to hold a license or an exemption. Exemptions are available for smaller generators that produce less than 50MW of power as these are considered to pose little risk to the network (Department of Electricity, 2001). Parties with an exemption are not subject to the balancing mechanism. This paper concerns those licensed parties signed up to the Balancing Settlement Code.

When there is an imbalance between a party's contracted volume and the amount of electricity actually delivered (metered volume) the cost of this imbalance is covered by two prices: The System Buy Price (SBP) and the System Sell Price (SSP). When a party under-generates they will have to buy the deficit at the SBP and when they over generate they will sell the surplus at the SSP (Elexon, 2014).

There are two different pricing methods used to calculate the SBP and SSP, the main pricing method and the reverse pricing method:

- $\quad$ The main pricing method is a volume weighted average of all imbalance cost experienced for the settlement period. 
- The reverse pricing method is calculated to reflect what a generator would have gotten if it had traded the imbalance at the power exchange.

For each settlement period only one imbalance direction is penalised. When there is too much power on the transmission system (Grid) it is considered long; the SSP is calculated using the main pricing method and the SBP is calculated by the reverse pricing method. Here there is no penalty for undergenerating as the party will be able to purchase energy at the same price as they traded at for their contracted volume. If they over-generate they will receive a lower price than what they could have obtained at the power exchange.

When the Grid is 'short' (not enough power) the SBP is calculated using the main pricing method and the SSP is calculated using the reverse pricing method. In this case the generator will receive the same price for excess generation as if it had been traded on the power exchange and the penalty for under-generating will be more severe: the generator will have to pay the SBP for the deficit, which will be higher than the price received for the contracted volume.

For a generator there are two likely conditions they will experience: they will either be in surplus, when they over generate; or in deficit, when they under generate. The different costs associated with these two conditions, surplus cost and deficit cost, are explained further in III.C.

A sample of the cost involved in trading for a single day is presented in Figure 1. Notice for a single settlement period only one cost is penalised while the other is zero, and that the direction of penalty can change from one period to the next.

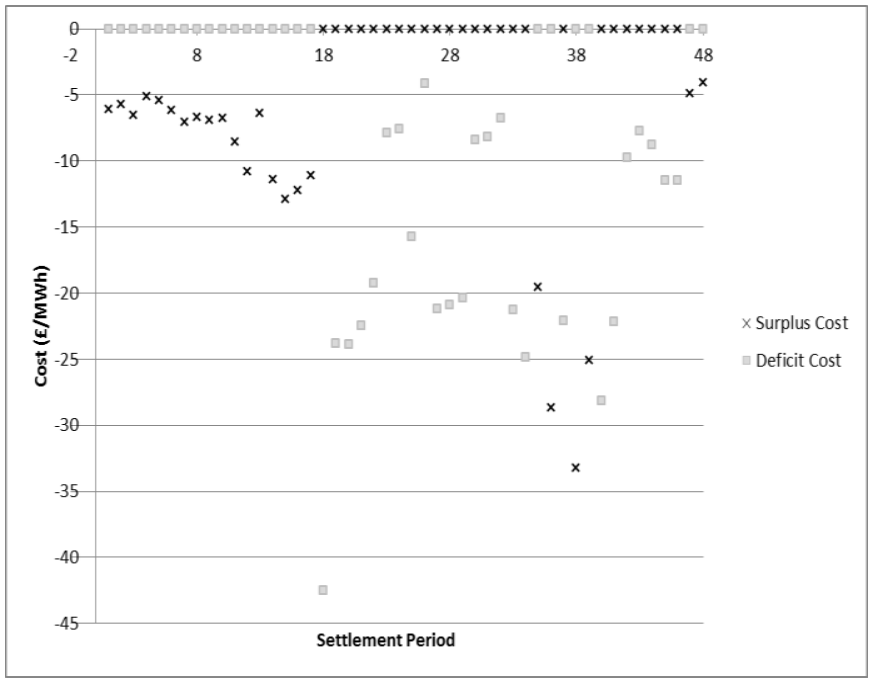

Figure 1: Example of surplus and deficit cost for the 21st Feb 2013. During any one settlement period only one cost is penalised.

When dealing with wind generation it can be assumed that there will be an imbalance between contracted and metered volume as even the best forecasting tools are not $100 \%$ accurate. Knowing there will be this imbalance, it would be financially beneficial if the imbalance is on the correct side of the market.
When the Grid is long there is no penalty for undergeneration so the preferred behaviour of the forecaster is to be optimistic in this situation. When the Grid is short, a more conservative forecast should be used as the penalties occur for under-generation.

\section{Methodology}

\section{A. Data}

The balancing mechanism prices used in this paper (System Sell Price and System Buy Price) were obtained from Elexon covering the year 2013 and the first three months of 2014. The market price, XP, is taken from the UKPX over the same period. This data set was split into a training set comprising all of 2013 and a validation set using the three months from 2014.

The wind data used is hourly averaged wind speeds taken from the FINO 1 weather station (Bundesministerium fuer Umwelt). One hourly data is used as a stand-in for actual wind speeds.

\section{B. Quantile Regression}

When a forecaster fits a linear regression model, it produces a forecast with an evenly distributed error; this means there is an even chance that the actual value will be above or below the predicted value (Draper \& Smith, 1998). In quantile regression (Koenker \& G. Bassett, 1978) this method is referred to as a $50^{\text {th }}$ quantile forecast, but when there is an asymmetric loss function, as there is in the case of the electricity market, this is not the most appropriate model.

Quantile regression is used in this research to form a probabilistic spread of forecasts that can be selected depending on the prediction of the Grid state. In Figure 2 the top line is the $90^{\text {th }}$ quantile, meaning that $90 \%$ of the time the actual value will be at or under the forecast, and bottom line is the $10^{\text {th }}$ quantile, meaning $10 \%$ of the time the actual value will be at or below the forecast.

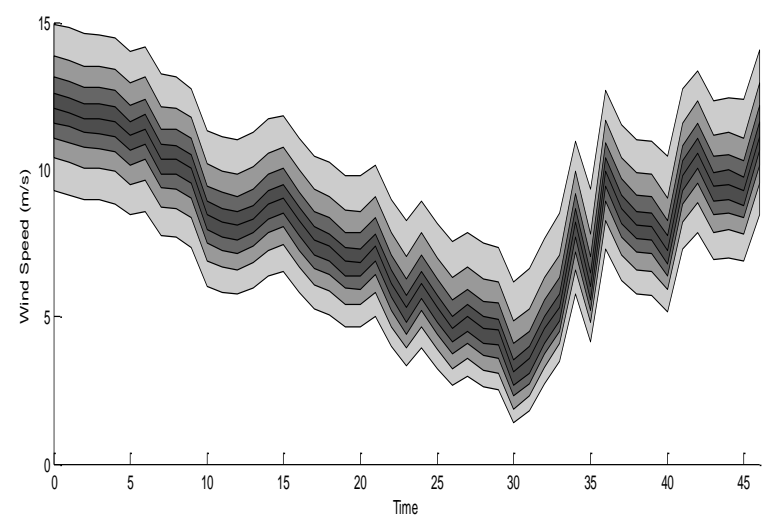

Figure 2: A series of quantile regressions can form a spread of possible forecasts for each time step.

The quantile regression models used in this paper were fitted using the "quantreg" package in $\mathrm{R}$ and the wind data 
was filtered using the methods outlined in (Hill, McMillan, Bell, \& Infield, 2012).

\section{Cost of Error Metric}

The cost of the error in a forecast is defined by three values: the SSP, SBP, and the exchange price received for the contracted volume (XP). This is an asymmetric loss function, albeit one that alternates. When a forecast over predicts, causing an under-generation, the loss is related to how much more is paid through the SBP to make up the deficit:

$$
\text { Deficit Cost }(£ / M W h)=X P-S B P
$$

When the Grid is long the deficit cost will be 0 .

When a forecast under predicts, the excess generation is sold at the SSP. This is viewed as a loss of potential revenue which could have been gained if this volume had been traded on the exchange:

$$
\text { Surplus Cost }(£ / M W h)=\text { SSP }- \text { XP }
$$

When the Grid is short the surplus cost will be 0 .

It was decided that the losses should be expressed as a negative number.

\section{Forecasting the Grid State}

Forecasting the state of the Grid requires the identification of the proportion of time the Grid is in each state. Overall in 2013 (the training set) the Grid was long $57.3 \%$, short $36.8 \%$ and neutral $5.9 \%$ of the time. A neutral Grid is a time when neither imbalance is penalised. The proportional share of each Grid state changes for each settlement period, with a higher percentage of neutral Grids found in the early settlement periods. The Grid is more likely to be long in the settlement period preceding an anticipated increase in demand like around settlement period 10 and 31 (Figure 3).

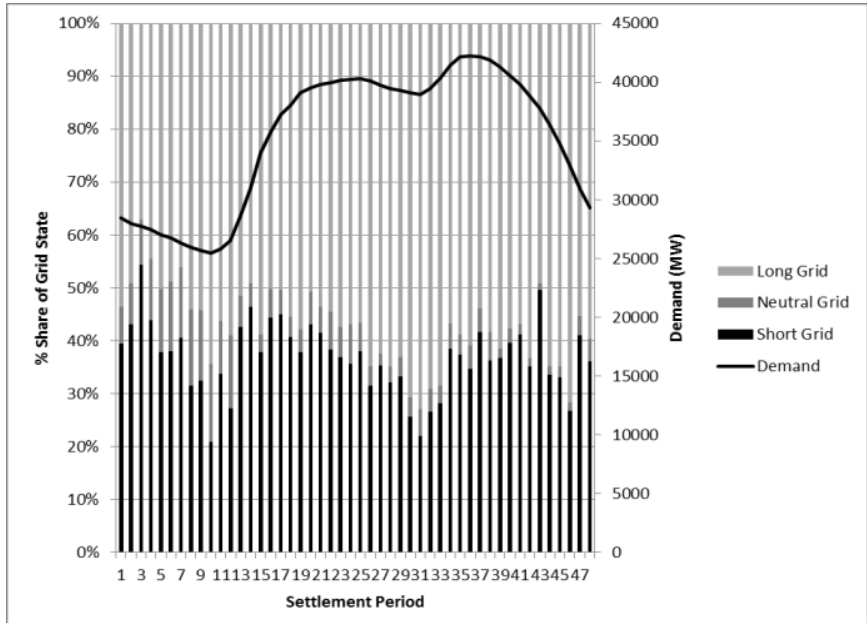

Figure 3: Share of Grid states for each settlement period and average demand.
The relationship between the settlement periods and probability of being in each Grid states is non-linear in nature. For this reason an Artificial Neural Network (ANN) (Picton, 1994) was chosen as the method to predict the Grid state. Along with the settlement period being predicted, inputs to the ANN are the current Grid state, the current imbalance prices (SBP, SSP) and the exchange price (XP). A short Grid is assigned a value of -1 , a long Grid a value of 1 and a neutral Grid 0 .

The ANN used is a Multilayer Perceptron with a single hidden layer of six nodes and is trained using back propagation. The market data from the year 2013 is used to train the ANN and the output is a numerical value. The interpretation of what output indicates a long or short Grid requires striking a balance between successful predictions and the number of settlement periods where no decision is made.

\section{E. Selecting the Quantile}

If the state of the Grid could be predicted perfectly each time then the optimal bidding strategy would be to contract 0 generation when the Grid is short, as the market price will be received for all generation produced, and to contract for full capacity when the Grid is long, as the deficit generation can be purchased at the same price it was sold. This strategy is expressed mathematically in (Jonsson, Pinson, Nielson, \& Madsen, 2014) based upon the expectation value for the deficit and surplus costs.

There are problems with this bidding strategy. Firstly no forecast is $100 \%$ accurate and the losses experienced when on the wrong side of the imbalance would counter the saving made when the prediction was correct. Secondly, the system operator has an expectation that the notifications of generation will reflect actual generation within an acceptable margin of error. Frequent and large deviations from contracted volume will draw the attention of the system operator who may chastise a party, ultimately revoking a party's license to trade if it deems the behaviour to jeopardise the stability of the Grid.

For these reasons when the Grid is forecast to be long the $80^{\text {th }}$ quantile is selected, instead of full production. When the Grid is short the $20^{\text {th }}$ quantile is used instead of zero. When it is a neutral Grid the $50^{\text {th }}$ quantile is used. The selection of the $80^{\text {th }}$ and $20^{\text {th }}$ quantile was an arbitrary choice to test the system. Future work will involve analysing the outputs from the ANN and the confidence levels associated with each output to better select the appropriate quantile.

\section{RESULTS}

\section{A. Assessing the model}

The model efficacy is assessed by the proportion of the data in which a quantile other than P50 is selected; the percentage of successful predictions forecasting a long or short Grid and the overall cost of operation as calculated using the error metric outlined in III.C. 


\section{B. Accuracy}

As a baseline of success a persistence style forecast of the market is used. A persistence forecast takes the current state of the market as the forecast. Using this method the state of the Grid was successfully predicted $65 \%$ of the time. Looking at the accuracy of the model for predicting whether the Grid is long or short; $69 \%$ of the long predictions were correct, and $59 \%$ of short predictions were correct.

To assess the efficacy of the ANN what is considered a forecast of a long/short Grid has to be defined. This could be done in a binary fashion by taking all positive outputs from the ANN to be a long prediction and any negative number as a short prediction. This produces the same overall accuracy as the persistence model $(65 \%)$. It performs worse for long predictions $(67 \%)$ and better for the short predictions $(60 \%)$ than persistence. This indicates that a binary approach to the classification of the Grid is not the optimal strategy and adding in a margin for the neutral Grid is required.

Appropriate boundaries for a long or short Grid are ascertained by varying the thresholds from 0.1 to 1 for the long prediction and -0.1 to -1 for the short prediction. When this was performed on the training set the thresholds that returned the lowest cost of operation were 0.3 for long and -0.1 for short. This limited the percentage of decisions made to $81 \%$ of the time series, but the accuracy of the forecaster improves to $67 \%$. This is due to an increase in accuracy to $70 \%$ for long predictions and $62 \%$ for short predictions. In this arrangement the Grid is forecast long $50 \%$ of the time and short $31 \%$. The $19.2 \%$ where the system forecasts a neutral Grid is well above the $5.9 \%$ observed in the training set. However, it is difficult to comment on whether this was the correct decision as the thresholds for a long or short Grid were determined by the values that returned the greatest saving in the training set. The percentage correct in Table I refers to times when the forecast was biased in the correct direction therefore using the Q50 is not considered correct or incorrect in this situation.

TABLE I. COST OF QUANTILE

\begin{tabular}{lcc}
\hline \hline Quantile (Q) & \% used & \% correct \\
\hline \hline Q80 & 49.6 & 70.0 \\
Q20 & 31.2 & 62.2 \\
Q50 & 19.2 & - \\
Overall & - & 67.0 \\
\hline
\end{tabular}

\section{Cost}

The wind speed forecasts and wind speed measurements are converted into power using a manufacturer's power curve for a $2.5 \mathrm{MW}$ wind turbine. The error from the forecast is expressed in terms of the cost accrued when the imbalance is handled by the balancing settlement code.

Table II summarises the cost associated with implementing each of the quantile regressions exclusively. An interesting observation is the best quantile to trade at was not the Q50 but the Q40. This may be particular to this data set but it is worth exploring the cost of trading based on each quantile further with a larger data set.

TABLE II. Cost OF QuANTILE

\begin{tabular}{ll}
\hline \hline Quantile (Q) & Cost $(\mathfrak{f})$ \\
\hline \hline 10 & -5580 \\
20 & -3895 \\
30 & -3359 \\
40 & $\mathbf{- 3 2 7 1}$ \\
50 & -3519 \\
60 & -3973 \\
70 & -4842 \\
80 & -6253 \\
90 & -8768 \\
\hline
\end{tabular}

This case study considered the power being traded from a single $2.5 \mathrm{MW}$ turbine over a three month period. The total cost of the error when using the Q50 forecast was $-£ 3519$. When the switching method was implemented the cost fell to -£2445, giving an overall saving of $£ 1074$.

\section{CONCLUSION AND DISCUSSION}

The intention of the method reported here is to provide a guide to interpreting a probabilistic forecast; the chosen model in this paper of constructing multiple quantile regressions is just one way to create a probabilistic spread. Any statistical forecast with a known variance could be used as a probabilistic forecast. Instead of disregarding the error for a point forecast, the error distribution can be used to form a probabilistic forecast.

The results achieved are quite promising with a saving of over $£ 1000$ for a single turbine in three months. Extending this to a full wind farm over a year will give a non-trivial saving in the operational cost of operating a wind farm. A reduction in the risks associated with trading may translate to a reduction in the price for wind power - a saving that can be passed onto customers.

\section{FUTURE WORK}

\section{A. Model Refinement}

The work carried out in this paper is a first attempt at adapting and applying the work of Jonsson et al. to the UK electricity market using a small market data set and an available wind data set as a proof of concept.

The work can be further refined with the acquisition of half hour averaged wind data from a site within the UK. The use of half hour wind data should improve the performance of all the forecasters used in this research. An improvement in forecaster performance will most likely reduce the overall savings reported here, as the cost associated with the Q50 will 
be lower when it is more accurate. However, this will give a better indication of the true model performance.

The model performance may be improved by the inclusion of Net Imbalance Volume, the total balancing required in a settlement period, and adding a second seasonal term to represent the variation across a week.

Currently the quantile choices are fixed at the $80^{\text {th }}, 20^{\text {th }}$, and $50^{\text {th }}$ quantile. The reason for this was for simplicity while the concept is tested but this can be refined. The output from the ANN is a range of values from -1 to 1 . By dividing these into suitable bins and determining the confidence level for each output the most suitable quantile can be selected on a period by period basis.

\section{B. Group Behaviour}

The current research involves simulating the trading of power from a single turbine, assuming it participates fully in the balancing mechanism. A single turbine should not affect the overall state of the Grid, but when the strategy is adopted by a large wind farm this could cause the Grid to invert. An investigation into this possibility would be needed before employing this in an industrial context.

\section{Modifications to the Balancing Settlement Code}

There is a modification to the balancing mechanism currently out for consultation (Elexon, 2015) that would replace the dual imbalance pricing with a single imbalance price that would reward generators that offset the imbalance of the system. Modifying the available data to simulate the proposed imbalance pricing method would allow for exploration of the consequences of a single imbalance price and the benefits of correctly predicting the state of the Grid.

\section{ACKNOWLEDGMENT}

For the data from the FINO project, we thank the BMWi (Bundesministerium fuer Wirtschaft und Energie, Federal Ministry for Economic Affairs and Energy) and the PTJ (Projekttraeger Juelich, project executing organisation).

This work has been funded by the EPSRC, project reference number EP/G037728/1.

\section{REFERENCES}

Bundesministerium fuer Umwelt, Federal Ministry for the Enviroment, Nature Conservation and Nuclear Safety, Germany, "FINO Project". Available: http://fino.bsh.de/

Draper, N. R., \& Smith, H. (1998). Applied Regression Analysis, 3rd ed. New York: John Wiley \& Sons.

Elexon. (2014, 06 10). Imbalance pricing guidance v7.0. Retrieved 11 11, 2014, from elexon.co.uk: http://www.elexon.co.uk/wpcontent/uploads/2014/06/imbalance_pricing_guidan ce_v7.0.pdf

Elexon. (2015, 01 15). https://www.elexon.co.uk/modproposal/p316/. Retrieved 01 15, 2015, from elexon.co.uk: https://www.elexon.co.uk/wpcontent/uploads/2014/11/230_17-P316-Initial-

Written-Assessment-v1.0.pdf

Galloway, S., Bell, G., McDonald, J., \& Siewierski, T. (2006). Managing the risk of trading wind energy in a competitive market. IEEE Proc.-Gener. Transm. Distrib, 106-114.

Gardner, P., \& Papadopoulos, I. (2012). The limiting factor for wind integration. Glasgow: GL Garrad Hassan.

Hill, D. C., McMillan, D., Bell, K. R., \& Infield, D. (2012). Application of Auto-Regressive Models to U.K.Wind Speed Data for Power System Impact Studies. IEEE TRANSACTIONS ON SUSTAINABLE ENERGY, 3(1), 134-141.

HM Government. (2001). The Electricity (class exemptions from the requirement for a licence) Order 2001. London.

Jonsson, T., Pinson, P., Nielson, H. A., \& Madsen, H. (2014). Exponential smoothing approaches for prediction in real time electricity markets. Energies, 3710-3732.

Koenker, R., \& G. Bassett, J. (1978, Jan). Regression Quantiles. Econometrica, 46(1), 33-50.

Matevosyan, J., \& Soder, L. (2006). Minimisation of imbalance cost trading wind power on the short term power market. IEEE Transactions on Power Systems.

Picton, P. (1994). Introduction to Neural Networks. Michigan: MacMillan Publishers Limited. 